\section{P132 MANAGEMENT OF SYMPTOMATIC MEN IN A LEVEL 2 SEXUAL HEALTH SERVICE}

doi:10.1136/sextrans-2012-050601c.132

N Dearing, ${ }^{*}$ M Pammi. Nottingham City Hospital, Nottingham, UK

Background We established a young persons level 2 community sexual health service (YP clinic) to encourage access to care. BASHH STI Management Standards warrants in-house microscopy for managing men with dysuria and/or urethral discharge symptoms not currently available at this clinic.

Aims To determine percentage of men presenting with dysuria and/or urethral discharge, rates of Chlamydia in men attending with and without these symptoms and number of untreated men after their clinic visit.

Methods Case notes of all new male heterosexual patient attendances at the YP clinic from 5 January 2011 to 23 October 2011 were reviewed. The management of symptomatic men were compared retrospectively with the outcome to look for missed/untreated STIs (sexually transmitted infections).

Results Total 91 attendances. 23 patients (25\%) were asymptomatic of which 5 tested positive for Chlamydia. Commonest symptoms were dysuria (29\%) rash/skin problem (25\%) or lumps (20\%). 10 patients presented with dysuria only, 1 tested positive for Chlamydia. 16 patients presented with discharge and dysuria, 5 tested positive for Chlamydia. In patients presenting with other symptoms $(46 \%)$ the following STIs were diagnosed: warts $n=15$, ulcers $\mathrm{n}=1$, scabies $\mathrm{n}=1$. Two patients tested positive for Chlamydia.

Discussion $43 \%$ of patients who did not report symptoms required treatment for an STI. Overall $23 \%$ of the dysuria and/or discharge group and $11 \%$ of patients without these symptoms tested positive for Chlamydia. $86 \%$ of the positive Chlamydia test results in the dysuria and/or discharge group had been treated in clinic compared with only $57 \%$ of positive Chlamydia results in the remaining patients.

Conclusion The absence of microscopy did not lead to more untreated Chlamydia infections in men with discharge and/or dysuria but denying symptomamtic young men the opportunity of being seen in the community may risk untreated infection, onward transmission and complication.

\section{P133 UNWANTED PREGNANCIES AFTER A GU CONSULTATION}

doi:10.1136/sextrans-2012-050601c.133

A De Burgh-Thomas, ${ }^{*}$ K Botly, G Morris. Gloucestershire Care Services, Gloucester, UK

Aims To assess the contraception element of the consultation in patients seen in our GU service.

Objectives To assess the proportion of patients attending for termination that had been seen in the GU service in the year prior. Establish how many of these patients were recorded as having inadequate contraception. The proportion of patients identified as needing enhanced contraception that received any. Establish the accuracy of the GU pregnancy risk assessment.

Methods The 1st arm reviewed the GU notes of the patients that had attended GU within a year of their termination. The 2 nd arm reviewed notes from 100 sequential females attending GUM.

Results 67 of $820(8.2 \%)$ women undergoing a TOP had attended GU in the previous year. Seven stated at consultation that they wanted to be pregnant, Three patients attended twice which gives a sample of 63. Retrospective assessment showed that in 8 (13.3.\%) pregnancy may have been avoided by intervention in GU. 41 women (41/63)(65.1\%) that went on to become pregnant and underwent a termination were identified at their GU visit as either not using any contraception or were relying on condoms that are known to be an inadequate contraceptive choice. This compared to $29 \%$ in the general audit of women attending GU. Despite identifying inadequate contraception enhanced contraception was not initiated in any of the patients that went on to become pregnant (1st arm) and in only 7\% of the general audit of GU attendees (2nd arm). Concordance between pregnancy assessment recorded in the notes and retrospectively assessed was 43\% (1st arm) and $62 \%$ (2nd arm) (see abstract P133 table 1).

Discussion We demonstrate that $93 \%$ of those identified as having urgent contraceptive needs leave without their needs having been addressed. We also demonstrate that contraception compliance is documented poorly. We will discuss changes that we have introduced to meet this service need.

Abstract P133 Table 1 Contraceptive data collection of BASHH auditable standards

\begin{tabular}{lllc}
\hline & BASHH standard & 1st arm & 2nd arm \\
\hline Date of last menstrual period & $100 \%$ & $89 \%$ & $90 \%$ \\
Contraception type recorded & $100 \%$ & $93 \%$ & $100 \%$ \\
Contraception compliance recorded & $100 \%$ & $31 \%$ & $41 \%$ \\
Pregnancy risk assessed & $100 \%$ & $68 \%$ & $79 \%$ \\
\hline
\end{tabular}

\section{P134 REACHING YOUTH WITH OUT-OF-FACILITY HIV AND REPRODUCTIVE HEALTH SERVICES}

doi:10.1136/sextrans-2012-050601c.134

${ }^{1} \mathrm{D}$ Denno, ${ }^{2} \mathrm{~V}$ Chandra-Mouli, ${ }^{3} \mathrm{M}$ Osman. ${ }^{*}$ University of Washington, Seattle, Washington, UK; ${ }^{2}$ World Health Organisation; ${ }^{3}$ University College London, London, UK

Background Many young people, particularly those who are marginalised and most at risk for HIV and reproductive health-related problems, cannot or will not seek traditional facility-based health services. Policies and programs are being implemented to provide them with these health services in the community. We sought to systematically review the effectiveness of such community-based HIV and reproductive health service policies and programs delivered via outreach on increasing health service utilisation among adolescents and young adults (ie, among 10-24 year olds).

Methods A systematic literature review of literature published prior to March 2010 was undertaken to identify policies promoting or programs delivering HIV or reproductive health services in the community. We reviewed studies that evaluated uptake of services or commodities. Data from studies meeting inclusion criteria were qualitatively analysed.

Results 20 studies met inclusion criteria, including 10 containing comparative data (eg, before and after study or control study design). The studies generally demonstrated positive impact, although results varied across settings and approaches. The most successful approaches included mail-based chlamydia screening in the Netherlands, condom distribution via street outreach in Louisiana, home-based HIV counselling and testing in Malawi, and promotion of over-the-counter access to emergency contraception in various countries.

Conclusions Overall, the studies suggest that out-of-facility approaches can be important avenues to reach marginalised youth. Rigorous implementation research is needed to assess the true impact that such strategies can have. Determining ways to effectively reach adolescents and young adults with HIV and reproductive health services is crucial in reducing not only youth morbidity and mortality but also in mitigating adult disease burden. 